\title{
Neutrosophic Bipolar Vague Resolvable and Neutrosophic Bipolar Vague Irresolvable Spaces
}

Princy $\mathrm{R}^{1}$ and Mohana $\mathrm{K}^{*, 2}$

Received: 12 May 2021/ Accepted: 14 June 2021/ Published online: 18 June 2021

(C)Sacred Heart Research Publications 2017

\begin{abstract}
In this paper the concepts of neutrosophic bipolar vague resolvable, neutrosophic bipolar vague irresolvable, neutrosophic bipolar vague open hereditarily irresolvable spaces, maximally neutrosophic bipolar vague irresolvable spaces and neutrosophic bipolar vague submaximal spaces are introduced. Also we study several interesting properties of the neutrosophic bipolar vague open hereditarily irresolvable spaces besides giving characterization of these spaces by means of somewhat neutrosophic bipolar vague continuous and somewhat neutrosophic bipolar vague open functions.

Key words: Neutrosophic Bipolar Vague Resolvable, Neutrosophic Bipolar Vague Irresolvable, Neutrosophic Bipolar Vague Submaximal, Neutrosophic Bipolar Vague Open Hereditarily Irresolvable Space, Somewhat Neutrosophic Bipolar Vague Continuous and Somewhat Neutrosophic Bipolar Vague Open Functions.
\end{abstract}

\section{Introduction}

The fuzzy concept had invaded almost all branches of mathematics ever since the introduction of fuzzy sets by L. A. Zadeh [17]. The theory of fuzzy topological space was introduced and developed by C. L. Chang [3] and since then various notions in classical topology have been extended to fuzzy topological space. In 1995, the denition of Smarandaches neutrosophic set, neutrosophic sets and neutrosophic logic have been useful in many real applications to handle improbability.

Neutrosophy is a branch of philosophy which studies the source, nature and scope of neutralities, as well as their interactions with different ideational scales [14]. The neutrosophic set uses one single value to indicate the truth-membership grade,

\footnotetext{
${ }^{1,2}$ Department of Mathematics, Nirmala College for Women, Coimbatore, India.

Email: 1 princy.pjs@gmail.com, *,2 riyaraju1116@gmail.com
} 
indeterminacy-membership degree and falsity membership grade of an element in the universe X. The conception of Neutrosophic Topological space was introduced by A.A.Salama and S.A.Alblowi [11].

Bipolar-valued fuzzy sets, which was introduced by Lee $[8,9]$ is an extension of fuzzy sets whose membership degree range is extended from the interval $[0,1]$ to $[-1,1]$. The membership degrees of the Bipolar valued fuzzy sets signify the degree of satisfaction to the property analogous to a fuzzy set and its counter-property in a bipolar valued fuzzy set, if the membership degree is 0 it means that the elements are unrelated to the corresponding property. Furthermore if the membership degree is on $(0,1]$ it indicates that the elements somewhat fulfil the property, and if the membership degree is on $[-1,0)$ it indicates that elements somewhat satisfy the entire counter property. After that, Deli et al. [4] announced the concept of bipolar neutrosophic sets, as an extension lead of neutrosophic sets.

Neutrosophic vague set is a combination of neutrosophic set and vague set which was well-defined by Shawkat Alkhazaleh [13]. Neutrosophic vague theory is a useful tool to practise incomplete, indeterminate and inconsistent information. Satham Hussain [12] introduced Neutrosophic bipolar vague sets. Mohana K and Princy R [10] have introduced Neutrosophic Bipolar Vague sets in topological spaces.

The concepts of resolvability and irresolvability in topological spaces was introduced by E. Hewit[7]. The concept of open hereditarily irresolvable spaces in the classical topology was introduced by A. Gelikin [6]. The concept of fuzzy resolvable and irresolvable spaces was introduced by G. Thangaraj and G. Balasubramanian[16]. In this paper the concept of neutrosophic bipolar vague resolvable, neutrosophic bipolar vague irresolvable, neutrosophic bipolar vague open hereditarily irresolvable spaces and maximally neutrosophic bipolar vague irresolvable spaces are introduced.

\section{Preliminaries}

Definition 2.1 [10] If $\mathrm{A}=$ $\left\{\left\langle x,\left[T_{A}^{-}, T_{A}^{+}\right]^{+},\left[I_{A}^{-}, I_{A}^{+}\right]^{+},\left[F_{A}^{-}, F_{A}^{+}\right]^{+},\left[T_{A}^{-}, T_{A}^{+}\right]^{-},\left[I_{A}^{-}, I_{A}^{+}\right]^{-},\left[F_{A}^{-}, F_{A}^{+}\right]^{-}\right\}\right\rangle$and $\mathrm{B}=$ $\left\{\left\langle x,\left[T_{B}^{-}, T_{B}^{+}\right]^{+},\left[I_{B}^{-}, I_{B}^{+}\right]^{+},\left[F_{B}^{-}, F_{B}^{+}\right]^{+},\left[T_{B}^{-}, T_{B}^{+}\right]^{-},\left[I_{B}^{-}, I_{B}^{+}\right]^{-},\left[F_{B}^{-}, F_{B}^{+}\right]^{-}\right\}\right\rangle$where $\left(T^{+}\right)^{+}$ $=1-\left(F^{-}\right)^{+},\left(F^{+}\right)^{+}=1-\left(T^{-}\right)^{+}$and $\left(T^{+}\right)^{-}=-1-\left(F^{-}\right)^{-},\left(F^{+}\right)^{-}=-1-\left(T^{-}\right)^{-}$. $T^{+}, I^{+}, F^{+}: X \rightarrow[0,1]$ and $T^{-}, I^{-}, F^{-}: X \rightarrow[-1,0]$ are the two neutrosophic bipolar vague sets then their union, intersection and complement are well-defined as follows:

1. $\mathrm{A} \cup \mathrm{B}=\left\{\max \left[T_{A}^{-}, T_{B}^{-}\right]^{+}, \max \left[T_{A}^{+}, T_{B}^{+}\right]^{+}\right.$, 
$\min \left[I_{A}^{-}, I_{B}^{-}\right]^{+}, \min \left[I_{A}^{+}, I_{B}^{+}\right]^{+}$,

$\min \left[F_{A}^{-}, F_{B}^{-}\right]^{+}, \min \left[F_{A}^{+}, F_{B}^{+}\right]^{+}$,

$\min \left[T_{A}^{-}, T_{B}^{-}\right]^{-}, \min \left[T_{A}^{+}, T_{B}^{+}\right]^{-}$,

$\max \left[I_{A}^{-}, I_{B}^{-}\right]^{-}, \max \left[I_{A}^{+}, I_{B}^{+}\right]^{-}$,

$\left.\max \left[F_{A}^{-}, F_{B}^{-}\right]^{-}, \max \left[F_{A}^{+}, F_{B}^{+}\right]^{-}\right\}$.

2. $\mathrm{A} \cap \mathrm{B}=\left\{\min \left[T_{A}^{-}, T_{B}^{-}\right]^{+}, \max \left[T_{A}^{+}, T_{B}^{+}\right]^{+}\right.$,

$\max \left[I_{A}^{-}, I_{B}^{-}\right]^{+}, \min \left[I_{A}^{+}, I_{B}^{+}\right]^{+}$,

$\max \left[F_{A}^{-}, F_{B}^{-}\right]^{+}, \min \left[F_{A}^{+}, F_{B}^{+}\right]^{+}$,

$\max \left[T_{A}^{-}, T_{B}^{-}\right]^{-}, \min \left[T_{A}^{+}, T_{B}^{+}\right]^{-}$,

$\min \left[I_{A}^{-}, I_{B}^{-}\right]^{-}, \max \left[I_{A}^{+}, I_{B}^{+}\right]^{-}$,

$\left.\min \left[F_{A}^{-}, F_{B}^{-}\right]^{-}, \max \left[F_{A}^{+}, F_{B}^{+}\right]^{-}\right\}$.

3. $A^{c}=\left\{\left[1-T_{A}^{+}\right]^{+},\left[1-T_{A}^{-}\right]^{+},\left[1-I_{A}^{+}\right]^{+},\left[1-I_{A}^{-}\right]^{+},\left[1-F_{A}^{+}\right]^{+},\left[1-F_{A}^{-}\right]^{+}\right.$, $\left.\left[-1-T_{A}^{+}\right]^{-},\left[-1-T_{A}^{-}\right]^{-},\left[-1-I_{A}^{+}\right]^{-},\left[-1-I_{A}^{-}\right]^{-},\left[-1-F_{A}^{+}\right]^{-},\left[-1-F_{A}^{-}\right]^{-}\right\}$.

Definition 2.2 10] Suppose A and B be two neutrosophic bipolar vague sets defined over a universe of disclosure $\mathrm{X}$. We say that $\mathrm{A} \subseteq \mathrm{B}$ if and only if $\left[T_{A}^{-} \leq T_{B}^{-}\right]^{+},\left[T_{A}^{+} \leq T_{B}^{+}\right]^{+},\left[I_{A}^{-} \geq I_{B}^{-}\right]^{+},\left[I_{A}^{+} \geq I_{B}^{+}\right]^{+},\left[F_{A}^{-} \geq F_{B}^{-}\right]^{+},\left[F_{A}^{+} \geq F_{B}^{+}\right]^{+}$, $\left[T_{A}^{-} \geq T_{B}^{-}\right]^{-},\left[T_{A}^{+} \geq T_{B}^{+}\right]^{-},\left[I_{A}^{-} \leq I_{B}^{-}\right]^{-},\left[I_{A}^{+} \leq I_{B}^{+}\right]^{-},\left[F_{A}^{-} \leq F_{B}^{-}\right]^{-},\left[F_{A}^{+} \leq F_{B}^{+}\right]^{-}$.

Definition 2.3 [10] A neutrosophic bipolar vague topology (in short NBVT)on a non-empty set $\mathrm{X}$ is a family $\tau$ of Neutrosophic bipolar vague set in $\mathrm{X}$ sustaining the following axioms: $1.0,1 \in \tau$.

2. $G_{1} \cap G_{2} \in \tau$ for any $G_{1}, G_{2} \in \tau$.

3. $\cup G_{i} \in \tau$ for any arbitrary family $\left\{G_{i}: G_{i} \in \tau, i \in I\right\}$.

Under such case the pair $(\mathrm{X}, \tau)$ is known as the neutrosophic bipolar vague topological space (In short NBVTS) and any NBVS in $(\mathrm{X}, \tau)$ is known as bipolar vague open set (In short NBVOS) in X . The complement of a neutrosophic bipolar vague open set $A^{c}$ in a neutrosophic bipolar vague topological space $(\mathrm{X}, \tau)$ is referred as a neutrosophic bipolar vague closed set(In short NBVCS) in (X, $\tau)$.

Definition 2.4 10 Let $A_{N B V}$ be a NBVS of the universe $\mathrm{U}$ where $\forall u_{i} \in U$, $\widehat{T}_{A N B V}^{P}(\mathrm{x})=[1,1] ; \widehat{I}_{A_{N B V}}^{P}(\mathrm{x})=[0,0] ; \widehat{F}_{A_{N B V}}^{P}(\mathrm{x})=[0,0] ; \widehat{T}_{A_{N B V}^{N}}^{N}(\mathrm{x})=[-1,-1] ;$ $\widehat{I}_{A_{N B V}^{N}}^{N}(\mathrm{x})=[0,0] ; \widehat{F}_{A N B V}^{N}(\mathrm{x})=[0,0]$.

Then $A_{N B V}$ is called unit NBVS $\left(1_{N B V}\right.$ in short), where $1 \leq i \leq n$.

Definition 2.5 [10] Let $A_{N B V}$ be a NBVS of the universe U where $\forall u_{i} \in U$, 
$\widehat{T}_{A_{N B V}}^{P}(\mathrm{x})=[0,0] ; \widehat{I}_{A_{N B V}}^{P}(\mathrm{x})=[1,1] ; \widehat{F}_{A_{N B V}}^{P}(\mathrm{x})=[1,1] ; \widehat{T}_{A_{N B V}}^{N}(\mathrm{x})=[0,0] ; \widehat{I}_{A_{N B V}}^{N}(\mathrm{x})$ $=[-1,-1] ; \widehat{F}_{A N B V}^{N}(\mathrm{x})=[-1,-1]$.

Then $A_{N B V}$ is called zero NBVS ( $0_{N B V}$ in short), where $1 \leq i \leq n$.

Definition 2.6 [10] Let A be a NBVS in a NBVTS (X, $\tau)$. The neutrosophic bipolar vague interior of A (In short NBVint(A)) and the neutrosophic bipolar vague closure of $\mathrm{A}$ (In short $\mathrm{NBVcl}(\mathrm{A})$ ) are defined by,

1. NBVint $(\mathrm{A})=\cup\{G / \mathrm{G}$ is a NBVOS in $\mathrm{X}$ and $\mathrm{G} \subseteq A\}$.

2. $\operatorname{NBVcl}(\mathrm{A})=\cap\{K / \mathrm{K}$ is a NBVCS in $\mathrm{X}$ and $\mathrm{A} \subseteq K\}$.

\section{Neutrosophic Bipolar Vague Resolvable and Irresolvable}

Definition 3.1 A neutrosophic bipolar vague set A in neutrosophic bipolar vague topological space $(\mathrm{X}, \mathrm{T})$ is called a neutrosophic bipolar vague dense if there exists no neutrosophic bipolar vague closed set $\mathrm{B}$ in $(\mathrm{X}, \mathrm{T})$ such that $\mathrm{A} \subset \mathrm{B} \subset 1_{\sim}$.

Definition 3.2 Let $(\mathrm{X}, \mathrm{T})$ be a neutrosophic bipolar vague topological space. $(\mathrm{X}, \mathrm{T})$ is called neutrosophic bipolar vague resolvable if there exists a neutrosophic bipolar vague dense set $\mathrm{A}$ in $(\mathrm{X}, \mathrm{T})$ such that $\operatorname{NBV} \operatorname{cl}\left(A^{c}\right)=1_{\sim}$. Otherwise $(\mathrm{X}, \mathrm{T})$ is called neutrosophic bipolar vague irresolvable.

Example 3.3 Let $X=\{a\}$. Define the neutrosophic bipolar vague sets A,B and C as follows:

$A=\langle x,[0.4,0.5][0.3,0.3][0.5,0.6][-0.6,-0.5][-0.4,-0.4][-0.5,-0.4]\rangle$,

$B=\langle x,[0.2,0.4][0.5,0.5][0.6,0.8][-0.7,-0.6][-0.5,-0.5][-0.4,-0.3]\rangle$ and

$C=\langle x,[0.3,0.5][0.4,0.4][0.5,0.7][-0.8,-0.4][-0.6,-0.6][-0.6,-0.2]\rangle$.

Clearly $T=\left\{0_{\sim}, 1_{\sim}, A\right\}$ is a neutrosophic bipolar vague topology on X. Thus (X,T) is a neutrosophic bipolar vague topological space. Now

$N B V \operatorname{int}(B)=0_{\sim}, N B V \operatorname{int}(C)=0_{\sim}, N B V \operatorname{int}\left(B^{c}\right)=0_{\sim}, N B V \operatorname{int}\left(C^{c}\right)=$

$0_{\sim}, N B V c l(B)=1_{\sim}, N B V c l(C)=1_{\sim}, N B V c l\left(B^{c}\right)=1_{\sim}, N B V c l\left(C^{c}\right)=1_{\sim}$. Hence

there exists a neutrosophic bipolar vague dense set $\mathrm{B}$ in $(\mathrm{X}, \mathrm{T})$, such that $N B \operatorname{Vint}\left(B^{c}\right)=0_{\sim}$. Hence the neutrosophic bipolar vague topological space $(\mathrm{X}, \mathrm{T})$ is called a neutrosophic bipolar vague resolvable.

Example 3.4 Let $X=\{a\}$. Define the neutrosophic bipolar vague sets A,B and C as follows: 
$A=\langle x,[0.4,0.6][0.6,0.6][0.4,0.6][-0.5,-0.3][-0.7,-0.7][-0.7,-0.5]\rangle$,

$B=\langle x,[0.5,0.7][0.3,0.3][0.3,0.5][-0.6,-0.7][-0.4,-0.4][-0.3,-0.4]\rangle$ and

$C=\langle x,[0.6,0.8][0.4,0.4][0.2,0.4][-0.6,-0.4][-0.5,-0.5][-0.6,-0.4]\rangle$.

Clearly $T=\left\{0_{\sim}, 1_{\sim}, A\right\}$ is a neutrosophic bipolar vague topology on X. Thus $(\mathrm{X}, \mathrm{T})$ is a neutrosophic bipolar vague topological space. Now

$N B V i n t(B)=A, N B V i n t(C)=A, N B V c l(B)=1, N B V c l(C)=1$. Thus B and $\mathrm{C}$ are neutrosophic bipolar vague dense set in $(\mathrm{X}, \mathrm{T})$ such that

$N B V c l\left(B^{c}\right)=A^{c}$ and $N B V \operatorname{cl}\left(C^{c}\right)=A^{c}$. Hence the neutrosophic bipolar vague topological space $(\mathrm{X}, \mathrm{T})$ is called a neutrosophic bipolar vague irresolvable.

Proposition 3.5 Let (X, T) be a neutrosophic bipolar vague topological space. $(\mathrm{X}, \mathrm{T})$ is a neutrosophic bipolar vague resolvable space if and only if (X,T) has a pair of neutrosophic bipolar vague dense set $A_{1}$ and $A_{2}$ such that $A_{1} \subseteq A_{2}^{c}$.

Proof: Let $(\mathrm{X}, \mathrm{T})$ be a neutrosophic bipolar vague topological space and $(\mathrm{X}, \mathrm{T})$ is a neutrosophic bipolar vague resolvable space. Suppose that for all neutrosophic bipolar vague dense sets $A_{i}$ and $A_{j}$, we have $A_{i} \nsubseteq A_{j}^{c}$. Then $A_{i} \supset A_{j}^{c}$. Then $\operatorname{NBV} c l\left(A_{i}\right) \supset \operatorname{NBV} c l\left(A_{j}^{c}\right)$ which implies that $1_{\sim} \supset \operatorname{NBV} c l\left(A_{j}^{c}\right)$ then $N B V \operatorname{cl}\left(A_{j}^{c}\right) \neq 1_{\sim}$. Also $A_{j} \supset A_{i}^{c}$, then $N B V \operatorname{cl}\left(A_{j}\right) \supset \operatorname{NBV} \operatorname{cl}\left(A_{i}^{c}\right)$ which implies that $1_{\sim} \supset \operatorname{NBV} \operatorname{cl}\left(A_{i}^{c}\right)$. Then $\operatorname{NBV} c l\left(A_{i}^{c}\right) \neq 1_{\sim}$. Hence $N B V c l\left(A_{i}\right)=1_{\sim}$, but $N B V \operatorname{cl}\left(A_{i}^{c}\right) \neq 1 \sim$ for all neutrosophic bipolar vague set $A_{i}$ in $(\mathrm{X}, \mathrm{T})$. Which is a contradiction. Hence $(\mathrm{X}, \mathrm{T})$ has a pair of neutrosophic bipolar vague dense set $A_{1}$ and $A_{2}$ such that $A_{1} \subseteq A_{2}^{c}$.

Converse, suppose that the neutrosophic bipolar vague topological space (X,T) has a pair of neutrosophic bipolar vague dense sets $A_{1}$ and $A_{2}$, such that $A_{1} \subseteq A_{2}^{c}$. Suppose that $(\mathrm{X}, \mathrm{T})$ is a neutrosophic bipolar vague irresolvable space. Then for all neutrosophic bipolar vague dense set $A_{1}$ and $A_{2}$ in $(\mathrm{X}, \mathrm{T})$, we have $N B V \operatorname{cl}\left(A_{1}^{c}\right) \neq 1_{\sim}$. Then $N B V \operatorname{cl}\left(A_{2}^{c}\right) \neq 1_{\sim}$ implies that there exists a neutrosophic bipolar vague closed set $\mathrm{B}$ in $(\mathrm{X}, \mathrm{T})$ such that $A_{2}^{c} \subset B \subset 1_{\sim}$. Then $A_{1} \subseteq A_{2}^{c} \subset B \subset 1_{\sim}$ implies that $A_{1} \subseteq B \subset 1_{\sim}$. Which is a contradiction. Hence $(\mathrm{X}, \mathrm{T})$ is a neutrosophic bipolar vague resolvable space.

Proposition 3.6 If $(\mathrm{X}, \mathrm{T})$ is a neutrosophic bipolar vague irresolvable iff $N B \operatorname{Vint}(A) \neq 0_{\sim}$ for all neutrosophic bipolar vague dense set $\mathrm{A}$ in $(\mathrm{X}, \mathrm{T})$.

Proof: Since $(\mathrm{X}, \mathrm{T})$ is a neutrosophic bipolar vague irresolvable space, for all neutrosophic bipolar vague dense set $\mathrm{A}$ in $(\mathrm{X}, \mathrm{T}), \operatorname{NBV} \operatorname{cl}\left(A^{c}\right) \neq 1_{\sim}$. Then $(N B \operatorname{Vint}(A))^{c} \neq 1_{\sim}$, which implies $N B \operatorname{Vint}(A) \neq 0_{\sim}$. 
Conversely, $N B \operatorname{Vint}(A) \neq 0_{\sim}$, for all neutrosophic bipolar vague dense set $\mathrm{A}$ in $(\mathrm{X}, \mathrm{T})$. Suppose that $(\mathrm{X}, \mathrm{T})$ is a neutrosophic bipolar vague resolvable. Then there exists a neutrosophic bipolar vague dense set $\mathrm{A}$ in $(\mathrm{X}, \mathrm{T})$, such that $N B V \operatorname{cl}\left(A^{c}\right)=1_{\sim}$ implies that $(N B V \operatorname{int}(A))^{c}=1_{\sim}$, implies $N B V \operatorname{int}(A)=0_{\sim}$. Which is a contradiction. Hence $(\mathrm{X}, \mathrm{T})$ is a neutrosophic bipolar vague irresolvable space.

Definition 3.7 A neutrosophic bipolar vague topological space (X,T) is called a neutrosophic bipolar vague submaximal space if each neutrosophic bipolar vague A in $(\mathrm{X}, \mathrm{T})$ such that $N B \operatorname{Vcl}(A)=1_{\sim}$, then $A \in T$.

Proposition 3.8 If the neutrosophic bipolar vague topological space $(\mathrm{X}, \mathrm{T})$ is neutrosophic bipolar vague submaximal, then $(\mathrm{X}, \mathrm{T})$ is neutrosophic bipolar vague irresolvable.

Proof: Let $(\mathrm{X}, \mathrm{T})$ be a neutrosophic bipolar vague submaximal space. Assume that $(\mathrm{X}, \mathrm{T})$ is a neutrosophic bipolar vague resolvable space. Let $\mathrm{A}$ be a neutrosophic bipolar vague dense set in $(\mathrm{X}, \mathrm{T})$. Then $N B V c l\left(A^{c}\right)=1_{\sim}$. Hence $(N B \operatorname{Vint}(A))^{c}=1_{\sim}$, implies $N B \operatorname{Vint}(A)=0_{\sim}$. Then $A \in T$. Which is a contradiction to neutrosophic bipolar vague submaximal space of $(\mathrm{X}, \mathrm{T})$. Hence $(\mathrm{X}, \mathrm{T})$ is a neutrosophic bipolar vague irresolvable space. The converse of Proposition 3.8 is not true. See Example 3.4.

Definition 3.9 A neutrosophic bipolar vague topological space (X,T) is called a maximal neutrosophic bipolar vague irresolvable space if $(X, T)$ is neutrosophic bipolar vague irresolvable and every neutrosophic bipolar vague dense set A of $(\mathrm{X}, \mathrm{T})$ is neutrosophic bipolar vague open.

Example 3.10 Let $X=\{a\}$. Define the neutrosophic bipolar vague sets A,B and $\mathrm{A} \cap \mathrm{B}$ and $\mathrm{A} \cup \mathrm{B}$ as follows:

$A=\langle x,[0.5,0.6][0.2,0.2][0.4,0.5][-0.5,-0.6][-0.3,-0.3][-0.4,-0.5]\rangle$, $B=\langle x,[0.3,0.4][0.6,0.6][0.6,0.7][-0.4,-0.3][-0.6,-0.6][-0.7,-0.6]\rangle$, $A \cap B=\langle x,[0.3,0.4][0.6,0.6][0.6,0.7][-0.4,-0.3][-0.6,-0.6][-0.7,-0.6]\rangle$ and $A \cup B=\langle x,[0.5,0.6][0.2,0.2][0.4,0.5][-0.5,-0.6][-0.3,-0.3][-0.4,-0.5]\rangle$. Clearly $T=\left\{0_{\sim}, 1_{\sim}, A, B, A \cap B, A \cup B\right\}$ is a neutrosophic bipolar vague topology on $\mathrm{X}$. Thus $(\mathrm{X}, \mathrm{T})$ is a neutrosophic bipolar vague topological space. Now 
$N B \operatorname{Vint}\left(A^{c}\right)=0_{\sim}, N B V \operatorname{int}\left(B^{c}\right)=\vee\left\{0_{\sim}, B, A \cap B\right\}=B, N B V \operatorname{int}\left((A \cup B)^{c}\right)=$ $0_{\sim}, N B V \operatorname{int}\left((A \cap B)^{c}\right)=\vee\left\{0_{\sim}, B, A \cap B\right\}=\operatorname{Band} N B V \operatorname{cl}(A)=1_{\sim}, N B V c l(B)=$ $B^{c}, N B V c l(A \cup B)=1_{\sim}, N B V c l(A \cap B)=B^{c}, N B V c l\left((A \cup B)^{c}\right)=$ $\wedge\left\{1_{\sim}, B^{c},(A \cup B)^{c},(A \cap B)^{c}\right\}=(A \cup B)^{c}, N B V c l\left(A^{c}\right)=$ $\wedge\left\{1_{\sim}, A^{c}, B^{c},(A \cup B)^{c},(A \cap B)^{c}\right\}=A^{c}, N B V c l\left(0_{\sim}\right)=1_{\sim}$. Thus neutrosophic bipolar vague dense set in $(\mathrm{X}, \mathrm{T})$ are $\mathrm{A}, \mathrm{A} \cup \mathrm{B}, 1_{\sim}$ are neutrosophic bipolar vague open in $(\mathrm{X}, \mathrm{T})$. Hence $(\mathrm{X}, \mathrm{T})$ is a neutrosophic bipolar vague irresolvable and every neutrosophic bipolar vague dense set of $(X, T)$ is neutrosophic bipolar vague open. Therefore $(\mathrm{X}, \mathrm{T})$ is a maximally neutrosophic bipolar vague irresolvable space.

\section{Neutrosophic Bipolar Vague Open Hereditarily Irresolvable}

Definition $4.1(\mathrm{X}, \mathrm{T})$ is said to neutrosophic bipolar vague open hereditarily irresolvable if $N B \operatorname{Vint}(N B V \operatorname{cl}(A)) \neq 0_{\sim}$ then $N B V \operatorname{int}(A) \neq 0_{\sim}$ for any neutrosophic bipolar vague set $\mathrm{A}$ in $(\mathrm{X}, \mathrm{T})$.

Example 4.2 Let $X=\{a\}$. Define the neutrosophic bipolar vague sets $A_{1}, A_{2}$ and $A_{3}$ as follows:

$A_{1}=\langle x,[0.2,0.5][0.6,0.6][0.5,0.8][-0.4,-0.2][-0.6,-0.6][-0.8,-0.6]\rangle$, $A_{2}=\langle x,[0.6,0.7][0.5,0.5][0.3,0.4][-0.5,-0.4][-0.5,-0.5][-0.6,-0.5]\rangle$ and $A_{3}=\langle x,[0.4,0.5][0.4,0.4][0.5,0.6][-0.5,-0.6][-0.5,-0.5][-0.4,-0.5]\rangle$.

Clearly, $T=\left\{0_{\sim}, 1_{\sim}, A_{1}, A_{2}\right\}$ is a neutrosophic bipolar vague topological space on $\mathrm{X}$. Thus $(\mathrm{X}, \mathrm{T})$ is a neutrosophic bipolar vague topological space. Now $N B V c l\left(A_{1}\right)=A_{1}^{c}, N B V c l\left(A_{2}\right)=1_{\sim}, N B V c l\left(A_{3}\right)=A_{1}$. Also $N B V \operatorname{int}\left(N B V \operatorname{cl}\left(A_{1}\right)\right)=N B V i n t\left(A_{1}^{c}\right)=A_{1} \neq 0_{\sim}$ and $N B V i n t\left(A_{1}\right)=A_{1} \neq 0_{\sim}, N B V i n t\left(N B V \operatorname{cl}\left(A_{2}\right)\right)=N B V i n t\left(1_{\sim}\right)=1_{\sim} \neq 0_{\sim}$ and $N B V \operatorname{int}\left(A_{2}\right)=A_{2} \neq 0_{\sim}, N B V \operatorname{int}\left(N B V \operatorname{cl}\left(A_{3}\right)\right)=N B V \operatorname{int}\left(A_{1}^{c}\right)=A_{1} \neq 0_{\sim}$ and $N B V i n t\left(A_{3}\right)=A_{1} \neq 0_{\sim}$ and $N B V i n t\left(N B V \operatorname{cl}\left(A_{3}^{c}\right)\right)=N B V \operatorname{int}\left(A_{1}^{c}\right)=A_{1} \neq 0_{\sim}$ and $N B V \operatorname{int}\left(A_{3}^{c}\right)=A_{1} \neq 0_{\sim}$. Hence if $N B V \operatorname{int}(N B V c l(A)) \neq 0 \sim$ then $N B \operatorname{Vint}(A) \neq 0_{\sim}$ for any nonzero neutrosophic bipolar vague set $\mathrm{A}$ in $(\mathrm{X}, \mathrm{T})$. Thus $(\mathrm{X}, \mathrm{T})$ is a neutrosophic bipolar vague open hereditarily irresolvable space.

Proposition $4.3(\mathrm{X}, \mathrm{T})$ be a neutrosophic bipolar vague topological space. If $(\mathrm{X}, \mathrm{T})$ is neutrosophic bipolar vague open hereditarily irresolvable then $(\mathrm{X}, \mathrm{T})$ is neutrosophic bipolar vague irresolvable.

Proof: Let A be a neutrosophic bipolar vague dense set in $(\mathrm{X}, \mathrm{T})$. Then 
$N B V \operatorname{cl}(A)=1_{\sim}$, which implies that $N B \operatorname{Vint}(N B V \operatorname{cl}(A))=1_{\sim} \neq 0_{\sim}$. Since $(\mathrm{X}, \mathrm{T})$ is neutrosophic bipolar vague open hereditarily irresolvable, we have $N B \operatorname{Vint}(A) \neq 0_{\sim}$. Therefore by Proposition 3.6, $N B \operatorname{Vint}(A) \neq 0_{\sim}$ for all neutrosophic bipolar vague dense set in $(\mathrm{X}, \mathrm{T})$, implies that $(\mathrm{X}, \mathrm{T})$ is neutrosophic bipolar vague irresolvable. The converse of the above proposition need not be true by the following example.

Example 4.4 Let $X=\{a\}$. Define the neutrosophic bipolar vague sets $A, \operatorname{BandC}$ as follows:

$A=\langle x,[0.4,0.6][0.6,0.6][0.4,0.6][-0.4,-0.3][-0.6,-0.6][-0.7,-0.6]\rangle$,

$B=\langle x,[0.5,0.7][0.5,0.5][0.3,0.5][-0.5,-0.6][-0.5,-0.5][-0.4,-0.5]\rangle$ and

$C=\langle x,[0.5,0.6][0.3,0.3][0.4,0.5][-0.5,-0.6][-0.3,-0.3][-0.4,-0.5]\rangle$.

Clearly, $T=\left\{0_{\sim}, 1_{\sim}, A, B\right\}$ is a neutrosophic bipolar vague topological space on $\mathrm{X}$. Thus $(\mathrm{X}, \mathrm{T})$ is a neutrosophic bipolar vague topological space. Now $\mathrm{C}$ and $1_{\sim}$ are neutrosophic bipolar vague dense sets in $(\mathrm{X}, \mathrm{T})$. Then $N B \operatorname{Vint}(C)=A \neq 0 \sim$ and $N B \operatorname{Vint}\left(1_{\sim}\right)=1_{\sim} \neq 0_{\sim}$. Hence $(\mathrm{X}, \mathrm{T})$ is a neutrosophic bipolar vague irresolvable space. But $N B \operatorname{Vint}\left(N B V \operatorname{cl}\left(C^{c}\right)\right)=N B \operatorname{Vint}\left(A^{c}\right)=A \neq 0_{\sim}$ and $N B \operatorname{Vint}\left(C^{c}\right)=0_{\sim}$. Therefore $(\mathrm{X}, \mathrm{T})$ is not a neutrosophic bipolar vague open hereditarily irresolvable space.

Proposition 4.5 Let $(\mathrm{X}, \mathrm{T})$ be a neutrosophic bipolar vague open hereditarily irresolvable. Then $N B \operatorname{Vint}(A) \nsubseteq(N B \operatorname{Vint}(B))^{c}$ for any two neutrosophic bipolar vague dense sets $\mathrm{A}$ and $\mathrm{B}$ in $(\mathrm{X}, \mathrm{T})$.

Proof: Let A and B be any two neutrosophic bipolar vague dense sets in (X,T). Then $N B V \operatorname{cl}(A)=1_{\sim}$ and $N B V \operatorname{cl}(B)=1_{\sim}$ implies that $N B V \operatorname{int}(N B V \operatorname{cl}(A)) \neq 0_{\sim}$ and $N B V i n t(N B V c l(B)) \neq 0_{\sim}$. Since $(\mathrm{X}, \mathrm{T})$ is neutrosophic bipolar vague open hereditarily irresolvable, $N B \operatorname{Vint}(A) \neq 0_{\sim}$ and $N B \operatorname{Vint}(B) \neq 0_{\sim}$. Hence by proposition 3.5, $\mathrm{A} \nsubseteq B^{c}$. Therefore $N B \operatorname{Vint}(A) \subseteq A \nsubseteq B^{c} \subseteq(N B \operatorname{Vint}(B))^{c}$. Hence we have $N B \operatorname{Vint}(A) \subseteq(N B V \operatorname{int}(B))^{c}$ for any two neutrosophic bipolar vague dense sets A and $\mathrm{B}$ in $(\mathrm{X}, \mathrm{T})$.

Proposition 4.6 Let $(\mathrm{X}, \mathrm{T})$ be a neutrosophic bipolar vague topological space. If $(\mathrm{X}, \mathrm{T})$ is neutrosophic bipolar vague open hereditarily irresolvable then $N B \operatorname{Vint}(A)=0_{\sim}$ for any nonzero neutrosophic bipolar vague dense set $\mathrm{A}$ in $(\mathrm{X}, \mathrm{T})$ implies that $N B \operatorname{Vint}(N B \operatorname{Vll}(A))=0_{\sim}$. 
Proof: Let A be a neutrosophic bipolar vague set in $(\mathrm{X}, \mathrm{T})$, such that $N B V \operatorname{int}(A)=0_{\sim}$. We claim that $N B V \operatorname{int}(N B V c l(A))=0_{\sim}$. Suppose that $N B \operatorname{Vint}(N B V \operatorname{cl}(A))=0_{\sim}$. Since $(\mathrm{X}, \mathrm{T})$ is neutrosophic bipolar vague open hereditarily irresolvable, we have $N B V \operatorname{int}(A) \neq 0_{\sim}$. Which is a contradiction to $N B \operatorname{Vint}(A)=0_{\sim}$. Hence $N B \operatorname{Vint}(N B V \operatorname{cl}(A))=0_{\sim}$.

Proposition 4.7 Let (X,T) be a neutrosophic bipolar vague topological space. If $(\mathrm{X}, \mathrm{T})$ is neutrosophic bipolar vague open hereditarily irresolvable then $N B V \operatorname{cl}(A)=1_{\sim}$ for any nonzero neutrosophic bipolar vague dense set $\mathrm{A}$ in $(\mathrm{X}, \mathrm{T})$ implies that $N B V \operatorname{cl}(N B \operatorname{Vint}(A))=1_{\sim}$

- Proof: Let A be a neutrosophic bipolar vague set in $(\mathrm{X}, \mathrm{T})$, such that $N B V \operatorname{cl}(A)=1_{\sim}$. Then we have $(N B V c l(A))^{c}=0_{\sim}$, which implies that $N B V \operatorname{int}\left(A^{c}\right)=0_{\sim}$. Since $(\mathrm{X}, \mathrm{T})$ is neutrosophic bipolar vague open hereditarily irresolvable by proposition 4.6. We have that $N B \operatorname{Vint}\left(N B V \operatorname{cl}\left(A^{c}\right)\right)=0_{\sim}$. Therefore $(N B V \operatorname{cl}(N B V \operatorname{int}(A)))^{c}=0_{\sim}$ implies $N B V \operatorname{cl}(N B V i n t(A))=1_{\sim}$.

\section{Somewhat Neutrosophic Bipolar Vague Continuous and Somewhat Neutrosophic Bipolar Vague Open}

Definition 5.1 Let $(\mathrm{X}, \mathrm{T})$ and $(\mathrm{Y}, \mathrm{S})$ be any two neutrosophic bipolar vague topological spaces. A function $f:(X, T) \rightarrow(Y, S)$ is called somewhat neutrosophic bipolar vague continuous if $A \in S$ and $f^{-1}(A) \neq 0_{\sim}$, then there exists a $B \in T$, such that $B \neq 0 \sim$ and $B \subseteq f^{-1}(A)$.

Definition 5.2 Let $(\mathrm{X}, \mathrm{T})$ and $(\mathrm{Y}, \mathrm{S})$ be any two neutrosophic bipolar vague topological spaces. A function $f:(X, T) \rightarrow(Y, S)$ is called somewhat neutrosophic bipolar vague open if $A \in T$ and $A \neq 0_{\sim}$, then there exists a $B \in S$, such that $B \neq 0 \sim$ and $B \subseteq f(A)$.

Proposition 5.3 Let $(\mathrm{X}, \mathrm{T})$ and $(\mathrm{Y}, \mathrm{S})$ be any two neutrosophic bipolar vague topological spaces. If the function $f:(X, T) \rightarrow(Y, S)$ is somewhat neutrosophic bipolar vague continuous and 1-1 and if $N B \operatorname{Vint}(A)=0_{\sim}$ for any nonzero neutrosophic bipolar vague set $\mathrm{A}$ in $(\mathrm{X}, \mathrm{T})$ then $N B \operatorname{Vint}(f(A))=0_{\sim} \operatorname{in}(Y, S)$.

Proof: Let A be a nonzero neutrosophic bipolar vague set in $(\mathrm{X}, \mathrm{T})$, such that $N B \operatorname{Vint}(A)=0_{\sim}$. To prove that $N B \operatorname{Vint}(f(A))=0_{\sim}$. Suppose that $N B \operatorname{Vint}(f(A)) \neq 0_{\sim}$ in $(\mathrm{Y}, \mathrm{S})$. Then there exists a nonzero neutrosophic bipolar 
vague set $\mathrm{B}$ in $(\mathrm{Y}, \mathrm{S})$, such that $B \subseteq f(A)$. Then $f^{-1}(B) \subseteq f^{-1}(f(A))$. Since $\mathrm{f}$ is somewhat neutrosophic bipolar vague continuous, there exists a $C \in T$, such that $C \neq 0_{\sim}$ and $C \subseteq f^{-1}(B)$. Hence $C \subseteq f^{-1}(B) \subseteq A$, which implies that $N B \operatorname{Vint}(A) \neq 0_{\sim}$. Which is a contradiction. Hence $N B \operatorname{Vint}(f(A))=0_{\sim}$ in $(\mathrm{Y}, \mathrm{S})$.

Proposition 5.4 Let $(\mathrm{X}, \mathrm{T})$ and $(\mathrm{Y}, \mathrm{S})$ be any two neutrosophic bipolar vague topological spaces. If the function $f:(X, T) \rightarrow(Y, S)$ is somewhat neutrosophic bipolar vague continuous and 1-1 and if $N B \operatorname{Vint}(N B V \operatorname{cl}(f(A)))=0_{\sim}$ for any nonzero neutrosophic bipolar vague set $\mathrm{A}$ in $(\mathrm{X}, \mathrm{T})$ then $N B V \operatorname{int}(N B V \operatorname{cl}(f(A)))=0 \sim$ in $(\mathrm{Y}, \mathrm{S})$.

Proof: Let A be a nonzero neutrosophic bipolar vague set in $(\mathrm{X}, \mathrm{T})$, such that $N B V \operatorname{int}(N B V \operatorname{cl}(A))=0_{\sim}$. We claim that $N B V \operatorname{int}(N B V \operatorname{cl}(f(A)))=0_{\sim}$ in $(\mathrm{Y}, \mathrm{S})$. Suppose that $N B V \operatorname{int}(N B V \operatorname{cl}(f(A))) \neq 0_{\sim}$ in $(\mathrm{Y}, \mathrm{S})$. Then $N B V c l(f(A)) \neq 0_{\sim}$. Then $(N B V \operatorname{cl}(f(A)))^{-1} \neq 0_{\sim}$. Now $(N B V \operatorname{cl}(f(A)))^{-1} \neq 0_{\sim} \in S$ and since $\mathrm{f}$ is somewhat neutrosophic bipolar vague continuous, there exists a $B \in T$, such that $B \neq 0_{\sim}$ and $B \subseteq f^{-1}\left((N B V c l(f(A)))^{-1}\right)$. That is $B \subseteq\left(f^{-1}(N B V c l(f(A)))\right)^{-1}$, which implies that $f^{-1}(N B V \operatorname{cl}(f(A))) \subseteq B^{-1}$. Since $\mathrm{f}$ is $1-1$, thus $A \subseteq f^{-1}(f(A)) \subseteq f^{-1}(N B V c l(f(A))) \subseteq B^{-1}$, implies that $A \subseteq B^{-1}$. Therefore $B \subseteq A^{-1}$ implies that $N B V \operatorname{int}\left(A^{c}\right) \neq 0_{\sim}$. Let $N B V \operatorname{int}\left(A^{c}\right)=C \neq 0_{\sim}$. Then we have $N B V \operatorname{cl}\left(N B \operatorname{Vint}\left(A^{-1}\right)\right)=N B V \operatorname{cl}(C) \neq 1_{\sim}$, implies that $N B \operatorname{Vint}(N B V \operatorname{cl}(A)) \neq 0_{\sim}$. Which is a contradiction. Hence $N B V \operatorname{int}(N B V \operatorname{cl}(f(A)))=0_{\sim}$ in $(\mathrm{Y}, \mathrm{S})$.

Proposition 5.5 Let $(\mathrm{X}, \mathrm{T})$ and $(\mathrm{Y}, \mathrm{S})$ be any two neutrosophic bipolar vague topological spaces. If the function $f:(X, T) \rightarrow(Y, S)$ is somewhat neutrosophic bipolar vague open and is $N B \operatorname{Vint}(A)=0_{\sim}$ for any nonzero neutrosophic bipolar vague set $\mathrm{A}$ in $(\mathrm{Y}, \mathrm{S})$ then $N B \operatorname{Vint}\left(f^{-1}(A)\right)=0_{\sim} i n(X, T)$.

Proof: Let A be a nonzero neutrosophic bipolar vague set in (Y,S), such that $N B \operatorname{Vint}(A)=0_{\sim}$. We claim that $N B \operatorname{Vint}\left(f^{-1}(A)\right)=0_{\sim}$ in $(\mathrm{X}, \mathrm{T})$. Suppose that $N B \operatorname{Vint}\left(f^{-1}(A)\right) \neq 0 \sim$ in $(\mathrm{X}, \mathrm{T})$. Then there exists a nonzero neutrosophic bipolar vague open set $\mathrm{B}$ in $(\mathrm{X}, \mathrm{T})$, such that $B \subseteq f^{-1}(A)$. Then we have $\mathrm{f}(\mathrm{B})$ $\subseteq f\left(f^{-1}(A)\right) \subseteq A$. Which implies that $f(B) \subseteq A$. Since $\mathrm{f}$ is somewhat neutrosophic bipolar vague open, there exists a $C \in S$, such that $C \neq 0_{\sim}$ and $C \subseteq f(B)$. Hence $C \subseteq f(B) \subseteq A$, which implies that $C \subseteq A$. Hence $N B \operatorname{Vint}(A) \neq 0_{\sim}$. Which is a contradiction. Hence $N B \operatorname{Vint}\left(f^{-1}(A)\right)=0_{\sim}$ in $(\mathrm{X}, \mathrm{T})$. 
Proposition 5.6 Let $(\mathrm{X}, \mathrm{T})$ and $(\mathrm{Y}, \mathrm{S})$ be any two neutrosophic bipolar vague topological spaces. Let $(\mathrm{X}, \mathrm{T})$ be a neutrosophic bipolar vague open hereditarily irresolvable space. If $f:(X, T) \rightarrow(Y, S)$ is somewhat neutrosophic bipolar vague open and somewhat neutrosophic bipolar vague continuous, 1-1 and onto function then $(\mathrm{Y}, \mathrm{S})$ is neutrosophic bipolar vague open hereditarily space.

Proof: Let A be a nonzero neutrosophic bipolar vague set in $(Y, S)$, such that $N B \operatorname{Vint}(A)=0_{\sim}$. Now $N B \operatorname{Vint}(A)=0_{\sim}$ and $\mathrm{f}$ is somewhat neutrosophic bipolar vague open implies that by proposition 5.5, $N B V \operatorname{int}\left(f^{-1}(A)\right)=0_{\sim}$ in $(\mathrm{X}, \mathrm{T})$. Since $(\mathrm{X}, \mathrm{T})$ is neutrosophic bipolar vague open hereditarily irresolvable space, we have $N B \operatorname{Vint}\left(N B V \operatorname{cl}\left(f^{-1}(A)\right)\right)=0_{\sim}$ in $(\mathrm{X}, \mathrm{T})$, by proposition 4.6. Since $N B V \operatorname{int}\left(N B V \operatorname{cl}\left(f^{-1}(A)\right)\right)=0 \sim$ and $\mathrm{f}$ is somewhat neutrosophic bipolar vague continuous by proposition 5.4, we have $N B \operatorname{Vint}\left(N B V c l\left(f\left(f^{-1}(A)\right)\right)\right)=0_{\sim}$. Since $\mathrm{f}$ is onto, thus $N B V \operatorname{int}(N B V \operatorname{cl}(A))=0_{\sim}$. Hence by proposition 4.6, (Y,S) is a neutrosophic bipolar vague open hereditarily irresolvable space.

\section{References}

[1] Atanassov. K., Intuitionistic fuzzy sets, Fuzzy Sets and Systems, 20 (1986).

[2] H.Bustince \& P.Burillo, Vague sets are intuitionistic fuzzy sets, Fuzzy sets and Systems, 79(3), 403 - 405 (1996).

[3] C. L. Chang, Fuzzy Topological Spaces, Journal of Mathematics Annals and Applications, 24, 182-190 (1968).

[4] Deli I, Ali M, Smarandache F, Bipolar neutrosophic sets and their application based on multi-criteria decision making problems., International conference on advanced mechatronic systems, 249 - 254 (2015).

[5] Gau. W. L, Buehrer. D.J., Vague sets, IEEE Transcations on Systems, Man and Cybernatics, 23(2), 610-614 (1993).

[6] A. Gelkin, Ultrafilters and decomposable spaces, Vestnik. Mosk. Univ. Math., 24(5), 51 (1969).

[7] E. Hewit, A Problem in set Theoretic Topology, Duke, Math. Journal, 10, 309-333 (1943).

[8] Lee. K.M, Comparison of interval-valued fizzy sets, intuitionistic fuzzy sets and bipolar-valued fuzzy sets, Journal of Fuzzy Logic Intelligent, 14(2), 125 - 129 (2004). 
ISSN: 2456-8686, 5(1), 2021:076-087

https://doi.org/10.26524/cm94

[9] Lee. K.M, Bipolar-valued fuzzy sets and their operations, Proc. International Conference on Intelligent Technologies, Bangkok, Thailand, 307 - 312 (2000).

[10] Mohana K , Princy R and Florentin Smarandache, An introduction to Neutrosophic Bipolar Vague topological spaces, Neutrosophic Sets and Systems, 29, 62 - 69 (2019).

[11] A.A.Salama and S.A.AL.Blowi, Neutrosophic Set and Neutrosophic Topological Spaces, IOSR Journal of Mathematics, 3(4), 31 -35 (2012).

[12] Satham Hussain S, Jahir Hussain R, Young Bae Jun, Florentin Smarandache, Neutrosophic Bipolar Vague Set and its application to Neutrosophic bipolar vague Graphs, Neutrosophic Sets and Systems, 28, 69 - 86 (2019).

[13] Shawkat Alkhazaleh, Neutrosophic vague set theory, Critical Review, 10, 29 39 (2015).

[14] F. Smarandache, a Unifying Field in Logics. Neutrosophy: Neutrosophic Probability, Set and Logic,Rehoboth: American Research Press, (1999).

[15] G. Thangaraj and G . Balasubramanian, On Somewhat Fuzzy Continuous Functions, Journal of Fuzzy Mathematics, 11(2), 725736 (2003).

[16] G. Thangaraj and G . Balasubramanian, On fuzzy resolvable and fuzzy irresolvable spaces,Fuzzy Sets Rough Sets and Multivalued Operations and Applications, 2(1), 173-180 (2009).

[17] Zadeh. L. A, Fuzzy Sets, Information and Control, 8, 338 - 353 (1965). 